\title{
TWO-DIMENSIONAL HYBRIDS WITH MIXED BOUNDARY VALUE PROBLEMS
}

\author{
Marzena Szajewska, Agnieszka Tereszkiewicz* \\ Institute of Mathematics, University of Bialystok, 1M Ciolkowskiego, PL-15-245 Bialystok, Poland \\ * corresponding author: a.tereszkiewicz@uwb.edu.pl
}

Abstract. Boundary value problems are considered on a simplex $F$ in the real Euclidean space $\mathbb{R}^{2}$. The recent discovery of new families of special functions, orthogonal on $F$, makes it possible to consider not only the Dirichlet or Neumann boundary value problems on $F$, but also the mixed boundary value problem which is a mixture of Dirichlet and Neumann type, ie. on some parts of the boundary of $F$ a Dirichlet condition is fulfilled and on the other Neumann's works.

KEYwORDS: hybrid functions, Dirichlet boundary value problem, Neumann boundary value problem, mixed boundary value problem.

\section{INTRODUCTION}

The boundary value problems, considered in this paper, occurring in a real Euclidean space $\mathbb{R}^{2}$ on finite region $F \subset \mathbb{R}^{2}$ that is half of a square or half of an equilateral triangle.

The main idea of this paper is to study the solutions of Helmholtz equation with the mixed boundary value problems. A surprising variety of recently emerged suitable new families of special functions makes that the realization of this idea is relatively simple and straightforward in any dimension. In addition to the classical boundary value problems of Dirichlet and Neumann type, the new functions, called 'hybrids' [6. 10, display properties at the boundary of $F$, on some parts of the boundary being Dirichlet's, while on the remaining ones Neumann's.

The boundary value conditions play an important role in describing the physical phenomena. They are used, inter alia, in the theory of elasticity, electrostatics and fluid mechanics [2, 4, 16].

In Section 2 we introduce some facts about Weyl groups $C_{2}$ and $G_{2}$. In Section 3 we show the exact formulas for four families of special functions for each of the group $C_{2}$ and $G_{2}$. The branching rules used to separate variables in Section 4 are described in details for example in the following papers [9, 11, 14. In Section 5 three types of boundary value problems are considered for four families of special functions described in Section 3 Although for the case $A_{1} \times A_{1}$, there is no hybrid functions, the mixed boundary value problem occurs. We present this case in details in Appendix.

\section{WEYL GROUP $C_{2}$ AND $G_{2}$}

In this section we recall certain facts about Weyl groups $C_{2}$ and $G_{2}$ [1, 3, 5]. We use four bases in $\mathbb{R}^{2}$, namely $e_{-}, \alpha_{-}, \check{\alpha}-$ and $\omega$ - basis. The first one, $e$-basis, is a natural basis for an Euclidean space. The simple root basis, $\alpha$-basis, exists for every finite group

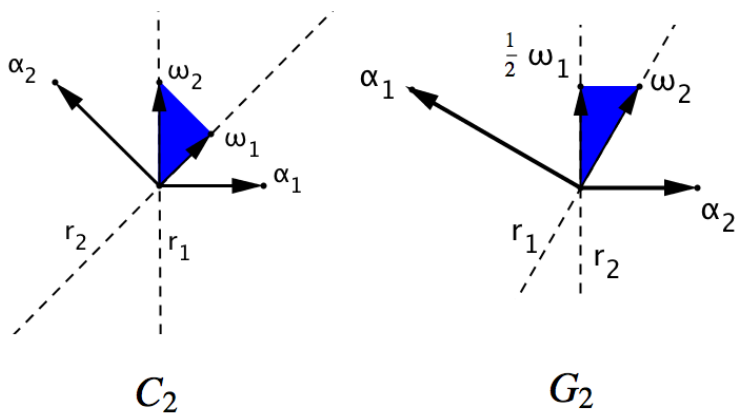

FiguRE 1. Shaded triangles represent the fundamental regions $F$ for $C_{2}$ and $G_{2}$ group.

generated by reflections. The co-root basis $\check{\alpha}$ is defined by the formula:

$$
\check{\alpha}_{i}=\frac{2 \alpha_{i}}{\left\langle\alpha_{i} \mid \alpha_{i}\right\rangle} .
$$

The $\omega$-basis is dual to simple root basis. The relationship between considered bases is standard for group theory and is expressed by:

$$
\left\langle\check{\alpha}_{i} \mid \omega_{j}\right\rangle=\delta_{i j}
$$

Below we present the $\alpha$-basis vectors in Cartesian coordinates for each of considered groups:

$$
\begin{aligned}
C_{2}: \alpha_{1}:=\frac{1}{\sqrt{2}}(1,-1)_{e}, & \alpha_{2}:=\frac{2}{\sqrt{2}}(0,1)_{e}, \\
G_{2}: \alpha_{1}:=(\sqrt{2}, 0)_{e}, & \alpha_{2}:=\left(-\frac{1}{\sqrt{2}}, \frac{1}{\sqrt{6}}\right)_{e} .
\end{aligned}
$$

The following notation for coordinates is used:

$$
\begin{gathered}
\mathbb{R}^{2} \ni \lambda=(a, b)_{\omega}=a \omega_{1}+b \omega_{2} . \\
\mathbb{R}^{2} \ni \boldsymbol{x}=\left(x_{1}, x_{2}\right)_{\check{\alpha}}=\left(y_{1}, y_{2}\right)_{e},
\end{gathered}
$$

where indexes $\omega, e$, and $\check{\alpha}$ denote $\omega$-, natural-, and ฉ̌-basis, respectively. 
The fundamental regions $F$ for $C_{2}$ and $G_{2}$ group, written in $\omega$-basis, have the vertices

$$
\begin{aligned}
& F_{C_{2}}=\left\{0, \omega_{1}, \omega_{2}\right\}, \\
& F_{G_{2}}=\left\{0, \frac{\omega_{1}}{2}, \omega_{2}\right\}
\end{aligned}
$$

and are shown in Figure 1

The groups $C_{2}$ and $G_{2}$ can be reduced to a subgroup $A_{1} \times A_{1}$ using a branching rule method described in [11, 14]. For $C_{2}$ case it is done by the projection matrix

$$
P_{C_{2}}=\left(\begin{array}{ll}
1 & 1 \\
0 & 1
\end{array}\right)
$$

acting on the whole orbit of a group. The branching rule is the following:

$$
O(a, b) \stackrel{P_{C_{2}}}{\longrightarrow} O(a+b) O(b) \cup O(b) O(a+b) .
$$

The reduction from $G_{2}$ to $A_{1} \times A_{1}$ is given by the matrix

$$
P_{G_{2}}=\left(\begin{array}{ll}
1 & 1 \\
3 & 1
\end{array}\right)
$$

The branching rule, in this case, has a form:

$$
\begin{aligned}
O(a, b) \stackrel{P_{G_{2}}}{\longrightarrow} & O(a+b) O(3 a+b) \\
\cup & O(2 a+b) O(b) \cup O(a) O(3 a+2 b) .
\end{aligned}
$$

For group $A_{1} \times A_{1}$ we use the following notation for coordinates

$$
\mathbb{R}^{2} \ni \boldsymbol{x}=(x, y)_{e} \in A_{1} \times A_{1} .
$$

\section{3. $C-, S_{-}, S^{s}-$, AND $S^{l}$-FUnCtions OF$$
G=C_{2} \text { OR } G_{2}
$$

The general formula for special functions corresponding to the Weyl group [5] is given by

$$
\sum_{w \in G} \sigma(w) e^{2 \pi i\langle w \lambda \mid \boldsymbol{x}\rangle},
$$

where the coordinates $\boldsymbol{x}=\left(x_{1}, x_{2}\right)_{\check{\alpha}} \in \mathbb{R}^{2}$ and weight $\lambda=(a, b)_{\omega}$ are given in $\check{\alpha}$ - and $\omega$-basis, respectively. The homomorphism $\sigma: G \rightarrow\{ \pm 1\}$ (by $G$ we denote the group $C_{2}$ or $G_{2}$ ) determine the four families of special functions 9], that are of interest to us. The map $\sigma(w)$ is a product of $\sigma\left(r_{l}\right), \sigma\left(r_{s}\right) \in\{ \pm 1\}$, where $r_{l}, r_{s}$ denote long and short reflections in $w$, respectively. Consequently, there are four types of homomorphisms $\sigma$ :

$$
\begin{aligned}
C: \sigma\left(r_{l}\right) & =\sigma\left(r_{s}\right)=1 \\
S: \sigma\left(r_{l}\right) & =\sigma\left(r_{s}\right)=-1 \\
S^{l}: \sigma\left(r_{l}\right) & =-1, \quad \sigma\left(r_{s}\right)=1 \\
S^{s}: \sigma\left(r_{l}\right) & =1, \quad \sigma\left(r_{s}\right)=-1
\end{aligned}
$$

\subsection{EXPLICIT FORMS OF $C$ - AND $S$-FUnCTIONS}

In this subsection we provide an exact formulas for the two types of special functions, namely, $C$ - and $S$-functions for $C_{2}$ and $G_{2}$ group. The upper signs in the formulas correspond to $C_{(a, b)}(\boldsymbol{x})$ functions and the lower ones match up to $S_{(a, b)}(\boldsymbol{x})$ functions [9, 13]:

$$
\begin{aligned}
& C_{2}: \pm 2\left[\cos \left(2 \pi\left((a+2 b) x_{1}+(-a-b) x_{2}\right)\right)\right. \\
& \quad \pm \cos \left(2 \pi\left((a+2 b) x_{1}-b x_{2}\right)\right) \\
& \quad+\cos \left(2 \pi\left(-a x_{1}+(a+b) x_{2}\right)\right) \\
& \quad+\cos \left(2 \pi\left(a x_{1}+b x_{2}\right)\right], \\
& G_{2}: 2\left[\operatorname { c o s } \left(2 \pi\left((2 a+b) x_{1}-(3 a+2 b) x_{2}\right)\right.\right. \\
& +\cos \left(2 \pi\left(a x_{1}+b x_{2}\right)\right. \\
& \quad \pm \cos \left(2 \pi(a+b) x_{1}-b x_{2}\right) \\
& \quad \pm \cos \left(2 \pi\left(a x_{1}-(3 a+b) x_{2}\right)\right. \\
& \quad \pm \cos \left(2 \pi\left((2 a+b) x_{1}-(3 a+b) x_{2}\right)\right. \\
& \left.+\cos \left(2 \pi(a+b) x_{1}-(3 a+2 b) x_{2}\right)\right] .
\end{aligned}
$$

3.2. ExpliCIT FORM OF $S^{s}$ AND $S^{l}$-FUnCtions Similarly, as in the previous subsection, we present exact formulas for $S^{l}$ - and $S^{s}$ - functions. Again, the upper signs correspond to $S_{(a, b)}^{s}(\boldsymbol{x})$ function and the lower ones belong to $S_{(a, b)}^{l}(\boldsymbol{x})$ function [9, 13]:

$$
\begin{gathered}
C_{2}: 2\left[\mp \cos \left(2 \pi\left((a+2 b) x_{1}-(a+b) x_{2}\right)\right)\right. \\
\quad \pm \cos \left(2 \pi\left((a+2 b) x_{1}-b x_{2}\right)\right) \\
-\cos \left(2 \pi\left(-a x_{1}+(a+b) x_{2}\right)\right) \\
\quad+\cos \left(2 \pi\left(a x_{1}+b x_{2}\right)\right], \\
G_{2}: 2 i\left[\sin \left(2 \pi\left((a+b) x_{1}-(3 a+2 b) x_{2}\right)\right)\right. \\
\quad+\sin \left(2 \pi\left(a x_{1}+b x_{2}\right)\right) \\
\pm \sin \left(2 \pi\left((2 a+b) x_{1}-(3 a+2 b) x_{2}\right)\right) \\
\mp \sin \left(2 \pi\left(a x_{1}-(3 a+b) x_{2}\right)\right) \\
-\sin \left(2 \pi\left((2 a+b) x_{1}-(3 a+b) x_{2}\right)\right) \\
\left.\mp \sin \left(2 \pi\left((a+b) x_{1}-b x_{2}\right)\right)\right] .
\end{gathered}
$$

Remark 1 . The weight coordinates $(a, b)_{\omega}$ for the four families of special functions are different, namely

$$
\begin{aligned}
& C_{(a, b)}(\boldsymbol{x}): a, b \in \mathbb{Z}^{\geq 0}, \\
& S_{(a, b)}(\boldsymbol{x}): a, b \in \mathbb{Z}^{>0}, \\
& S_{(a, b)}^{s}(\boldsymbol{x}): \begin{cases}a \in \mathbb{Z}^{>0}, b \in \mathbb{Z}^{\geq 0} & \text { for } C_{2}, \\
a \in \mathbb{Z}^{\geq 0}, b \in \mathbb{Z}^{>0} & \text { for } G_{2},\end{cases} \\
& S_{(a, b)}^{l}(\boldsymbol{x}): \begin{cases}a \in \mathbb{Z}^{\geq 0}, b \in \mathbb{Z}^{>0} & \text { for } C_{2}, \\
a \in \mathbb{Z}^{>0}, b \in \mathbb{Z}^{\geq 0} & \text { for } G_{2},\end{cases}
\end{aligned}
$$

The next remark is a consequence of explicit forms of functions written in Subsections 3.1, 3.2

Remark 2. Four families of special functions are real in case of $C_{2}$ group.

The functions $C$-, $S$ - are real, and $S^{l}-, S^{s}$ - are pure imaginary in case of $G_{2}$ group. 


\section{Helmholtz differential EQUATION}

In this section we consider the well-known partial differential equation

$\Delta \Psi(\boldsymbol{x})=-w^{2} \Psi(\boldsymbol{x}), \quad w-$ positive real constant

called homogeneous Helmholtz equation (see for example [7, 8, 15] and references therein), where $\boldsymbol{x}=$ $\left(y_{1}, y_{2}\right)_{e}$ and

$$
\Delta=\frac{\partial^{2}}{\partial y_{1}^{2}}+\frac{\partial^{2}}{\partial y_{2}^{2}}
$$

Remark 3 [5. The special functions described in the previous section are eigenfunctions of the Laplace operator.

The explicit form of the Laplace operator in coordinates relative to the $\omega$-basis and $\check{\alpha}$-basis is the following

$$
\begin{aligned}
C_{2}: \Delta_{\omega} & =2 \partial_{1}^{2}-2 \partial_{1} \partial_{2}+\partial_{2}^{2}, \\
\Delta_{\check{\alpha}} & =\frac{1}{2} \partial_{1}^{2}+\partial_{1} \partial_{2}+\partial_{2}^{2}, \\
G_{2}: \Delta_{\omega} & =\partial_{1}^{2}-3 \partial_{1} \partial_{2}+3 \partial_{2}^{2}, \\
\Delta_{\check{\alpha}} & =2 \partial_{1}^{2}+2 \partial_{1} \partial_{2}+\frac{2}{3} \partial_{2}^{2} .
\end{aligned}
$$

Since

$$
\Delta e^{2 \pi i\langle\lambda \mid \boldsymbol{x}\rangle}=-4 \pi^{2}\langle\lambda \mid \lambda\rangle e^{2 \pi i\langle\lambda \mid \boldsymbol{x}\rangle}
$$

then we have

$$
\Delta \Psi_{\lambda}(\boldsymbol{x})=-4 \pi^{2}\langle\lambda \mid \lambda\rangle \Psi_{\lambda}(\boldsymbol{x}),
$$

where $\Psi_{\lambda}(\boldsymbol{x})$ is one of the functions $C, S, S^{s}$ or $S^{l}$. The inner product of $\lambda \mathrm{s}$ is equal

$$
\begin{aligned}
& C_{2}:\langle\lambda \mid \lambda\rangle=\frac{1}{2} a^{2}+a b+b^{2}, \\
& G_{2}:\langle\lambda \mid \lambda\rangle=2 a^{2}+2 a b+\frac{2}{3} b^{2} .
\end{aligned}
$$

\subsection{Separation of VARiables for the Helmholtz EQUATION}

Using a standard method of separation of variables for the Helmholtz equation 7

$$
\Delta \Psi(\boldsymbol{x})=-w^{2} \Psi(\boldsymbol{x}), \quad \boldsymbol{x}=\left(y_{1}, y_{2}\right)_{e},
$$

and searching for the solutions in the form $\Psi(\boldsymbol{x})=$ $X\left(y_{1}\right) Y\left(y_{2}\right)$, we have the following differential equation

$$
X^{\prime \prime} Y+X Y^{\prime \prime}+w^{2} X Y=0 .
$$

Introducing $-k^{2}$-separation constant, we get a pair of the ordinary differential equations easy to solve:

$$
X^{\prime \prime}+k^{2} X=0, \quad Y^{\prime \prime}+\left(w^{2}-k^{2}\right) Y=0 .
$$

A basic solution of (5) we can write in the form

$$
\begin{aligned}
& X_{1}\left(y_{1}\right)=\cos k y_{1}, \quad Y_{1}\left(y_{2}\right)=\cos \sqrt{w^{2}-k^{2}} y_{2}, \\
& X_{2}\left(y_{1}\right)=\sin k y_{1}, \quad Y_{2}\left(y_{2}\right)=\sin \sqrt{w^{2}-k^{2}} y_{2},
\end{aligned}
$$

where $k \neq 0$ and $w^{2}-k^{2} \neq 0$.

According to the assumptions that $k \neq 0$ and $w^{2} \neq$ $k^{2}$ we consider $C_{-}, S$-, $S^{s_{-}}$, and $S^{l}$-functions only with positive weights.

\section{2. $C_{2}$ CASE}

From the projection matrix $P_{C_{2}}$ (1) and the branching rule (2) we find two separation constants $-k_{1}^{2}$ and $-k_{2}^{2}$, which have a form

$$
\begin{array}{ll}
-k_{1}^{2}=-2(a+b)^{2} \pi^{2}, & w^{2}-k_{1}^{2}=2 b^{2} \pi^{2}, \\
-k_{2}^{2}=-2 b^{2} \pi^{2}, & w^{2}-k_{2}^{2}=2(a+b)^{2} \pi^{2} .
\end{array}
$$

Noting that $k_{1}^{2}=w^{2}-k_{2}^{2}$, as a separation constant we take

$$
-k^{2}=-2(a+b)^{2} \pi^{2}, \quad w^{2}-k^{2}=2 b^{2} \pi^{2} .
$$

Using the branching rule (2) from Section 2 for special functions $C, S, S^{s}, S^{l}$ we can rewrite those functions in the form:

$$
\begin{aligned}
C_{a, b}(\boldsymbol{x})=4[ & \cos \left(k y_{1}\right) \cos \left(\sqrt{w^{2}-k^{2}} y_{2}\right) \\
& \left.+\cos \left(\sqrt{w^{2}-k^{2}} y_{1}\right) \cos \left(k y_{2}\right)\right], \\
S_{a, b}(\boldsymbol{x})=4[ & \sin \left(\sqrt{w^{2}-k^{2}} y_{1}\right) \sin \left(k y_{2}\right) \\
& \left.-\sin \left(k y_{1}\right) \sin \left(\sqrt{w^{2}-k^{2}} y_{2}\right)\right], \\
S_{a, b}^{s}(\boldsymbol{x})=4[ & \cos \left(k y_{1}\right) \cos \left(\sqrt{w^{2}-k^{2}} y_{2}\right) \\
& \left.-\cos \left(\sqrt{w^{2}-k^{2}} y_{1}\right) \cos \left(k y_{2}\right)\right], \\
S_{a, b}^{l}(\boldsymbol{x})=-4 & {\left[\sin \left(\sqrt{w^{2}-k^{2}} y_{1}\right) \sin \left(k y_{2}\right)\right.} \\
& \left.+\sin \left(k y_{1}\right) \sin \left(\sqrt{w^{2}-k^{2}} y_{2}\right)\right] .
\end{aligned}
$$

By changing the variables by $y_{1}=\sqrt{2} x, y_{2}=\sqrt{2}$ we get the reduction to $A_{1} \times A_{1}$ subgroup

$$
\begin{aligned}
& C_{a, b}(\boldsymbol{x})=C_{a+b}(x) C_{b}(y)+C_{b}(x) C_{a+b}(y), \\
& S_{a, b}(\boldsymbol{x})=S_{a+b}(x) S_{b}(y)-S_{b}(x) S_{a+b}(y), \\
& S_{a, b}^{s}(\boldsymbol{x})=S_{a+b}(x) S_{b}(y)+S_{b}(x) S_{a+b}(y), \\
& S_{a, b}^{l}(\boldsymbol{x})=C_{a+b}(x) C_{b}(y)-C_{b}(x) C_{a+b}(y),
\end{aligned}
$$

The functions $C_{\mu}(x)$, and $S_{\mu}(x)$, on the right side of (6), are defined in Appendix. The coordinates $(x, y) \in A_{1} \times A_{1}$ are written in $\alpha$-basis.

\section{3. $G_{2}$ CASE}

From the projection matrix $P_{G_{2}}(3)$ and the branching rule (4) we find three separation constants $-k_{1}^{2},-k_{2}^{2}$, and $-k_{3}^{2}$, which have a form

$$
\begin{array}{ll}
-k_{1}^{2}=-2(2 a+b)^{2} \pi^{2}, & w^{2}-k_{1}^{2}=\frac{2}{3} b^{2} \pi^{2}, \\
-k_{2}^{2}=-2(a+b)^{2} \pi^{2}, & w^{2}-k_{2}^{2}=\frac{2}{3}(3 a+b)^{2} \pi^{2}, \\
-k_{3}^{2}=-2 a^{2} \pi^{2}, & w^{2}-k_{3}^{2}=\frac{2}{3}(3 a+2 b)^{2} \pi^{2} .
\end{array}
$$

Using the branching rule (4) from Section 2 for special functions $C, S, S^{s}, S^{l}$ we can rewrite those functions 


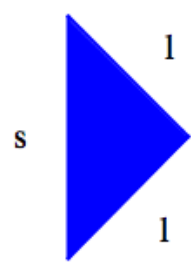

$C_{2}$

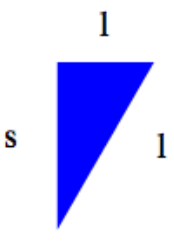

$G_{2}$
Figure 2. Fundamental regions of $C_{2}$ and $G_{2}$ groups. Sides are marked by $s$ and $l$ symbols which correspond to the reflection orthogonal to the short and long root, respectively.

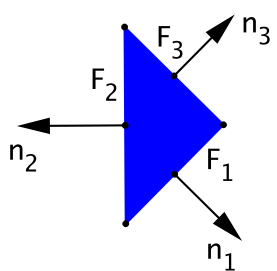

FiguRE 3 . The normal vectors and boundaries are indicated for the Weyl group $C_{2}$.

in the form:

$$
\begin{aligned}
C_{a, b}(\boldsymbol{x})=4[ & \cos \left(k_{1} y_{1}\right) \cos \left(\sqrt{w^{2}-k_{1}^{2}} y_{2}\right) \\
& +\cos \left(k_{2} y_{1}\right) \cos \left(\sqrt{w^{2}-k_{2}^{2}} y_{2}\right) \\
& \left.+\cos \left(k_{3} y_{1}\right) \cos \left(\sqrt{w^{2}-k_{3}^{2}} y_{2}\right)\right], \\
S_{a, b}(\boldsymbol{x})=4[ & -\sin \left(k_{1} y_{1}\right) \sin \left(\sqrt{w^{2}-k_{1}^{2}} y_{2}\right) \\
& +\sin \left(k_{2} y_{1}\right) \sin \left(\sqrt{w^{2}-k_{2}^{2}} y_{2}\right) \\
& \left.-\sin \left(k_{3} y_{2}\right) \sin \left(\sqrt{w^{2}-k_{3}^{2}} y_{2}\right)\right], \\
S_{a, b}^{s}(\boldsymbol{x})=4 i[ & -\cos \left(k_{1} y_{1}\right) \sin \left(\sqrt{w^{2}-k_{1}^{2}} y_{2}\right) \\
& -\cos \left(k_{2} y_{1}\right) \sin \left(\sqrt{w^{2}-k_{2}^{2}} y_{2}\right) \\
& \left.+\cos \left(k_{3} y_{1}\right) \sin \left(\sqrt{w^{2}-k_{3}^{2}} y_{2}\right)\right], \\
S_{a, b}^{l}(\boldsymbol{x})=4 i & -\sin \left(k_{1} y_{1}\right) \cos \left(\sqrt{w^{2}-k_{1}^{2}} y_{2}\right) \\
& +\sin \left(k_{2} y_{1}\right) \cos \left(\sqrt{w^{2}-k_{2}^{2}} y_{2}\right) \\
& \left.+\sin \left(k_{3} y_{1}\right) \cos \left(\sqrt{w^{2}-k_{3}^{2}} y_{2}\right)\right] .
\end{aligned}
$$

\begin{tabular}{|c|c|c|c|}
\hline \multirow[b]{2}{*}{$C_{2}, G_{2}$} & \multicolumn{3}{|c|}{ (D) $\quad \mathrm{N}$} \\
\hline & s $\quad 1$ & $\mathrm{~s}$ & 1 \\
\hline$C_{a, b}(\boldsymbol{x})$ & * * & 0 & 0 \\
\hline \multirow[t]{3}{*}{$S_{a, b}(\boldsymbol{x})$} & 0 & $*$ & $*$ \\
\hline & \multicolumn{3}{|c|}{$\mathrm{M}$} \\
\hline & $\mathrm{D}$ & $\sqrt{\Lambda}$ & N) \\
\hline$C_{2}, G_{2}$ & s $\quad 1$ & $\mathrm{~s}$ & 1 \\
\hline$S_{a, b}^{s}(\boldsymbol{x})$ & $0 \quad *$ & $*$ & 0 \\
\hline$S_{a, b}^{l}(\boldsymbol{x})$ & * 0 & 0 & * \\
\hline
\end{tabular}

By changing the variables by $y_{1}=\sqrt{2} x, y_{2}=\sqrt{6}$ we get the reduction to $A_{1} \times A_{1}$ subgroup

$$
\begin{aligned}
C_{a, b}(\boldsymbol{x})= & C_{a}(x) C_{3 a+2 b}(y) \\
& +C_{a+b}(x) C_{3 a+b}(y)+C_{2 a+b}(x) C_{b}(y), \\
S_{a, b}(\boldsymbol{x})= & S_{a}(x) S_{3 a+2 b}(y) \\
& -S_{a+b}(x) S_{3 a+b}(y)+S_{2 a+b}(x) S_{b}(y), \\
S_{a, b}^{s}(\boldsymbol{x})= & C_{a}(x) S_{3 a+2 b}(y) \\
& -C_{a+b}(x) S_{3 a+b}(y)-C_{2 a+b}(x) S_{b}(y), \\
S_{a, b}^{l}(\boldsymbol{x})= & S_{a}(x) C_{3 a+2 b}(y) \\
& -S_{a+b}(x) C_{3 a+b}(y)+S_{2 a+b}(x) C_{b}(y) .
\end{aligned}
$$

TABle 1. Behaviour of the functions $C, S, S^{s}$ and $S^{l}$ on the boundary $\partial F$ for $C_{2}$ and $G_{2}$ group where * denotes any function non-equivalent to 0 .

The functions $C_{\mu}(x)$, and $S_{\mu}(x)$, on the right side of (8), are defined in Appendix. The coordinates $(x, y) \in A_{1} \times A_{1}$ are written in $\alpha$-basis.

Proposition 1. $C_{\mu}(x)$, and $S_{\mu}(x)$ functions presented in (8) fulfill the following relationships

$$
\begin{gathered}
-k_{3} S_{3 a+2 b}(x) C_{a}(x) \\
+k_{2} S_{3 a+b}(x) C_{a+b}(x)-k_{1} S_{b}(x) C_{2 a+b}(x) \\
=\sqrt{3}\left(\sqrt{w^{2}-k_{3}^{2}} C_{3 a+2 b}(x) S_{a}(x)\right. \\
-\sqrt{w^{2}-k_{2}^{2}} C_{3 a+b}(x) S_{a+b}(x) \\
\left.-\sqrt{w^{2}-k_{1}^{2}} C_{b}(x) S_{2 a+b}(x)\right), \\
-k_{3} S_{3 a+2 b}(x) S_{a}(x) \\
+k_{2} S_{3 a+b}(x) S_{a+b}(x)+k_{1} S_{b}(x) S_{2 a+b}(x) \\
=\sqrt{3}\left(\sqrt{w^{2}-k_{3}^{2}} C_{3 a+2 b}(x) C_{a}(x)\right. \\
-\sqrt{w^{2}-k_{2}^{2}} C_{3 a+b}(x) C_{a+b}(x) \\
\left.-\sqrt{w^{2}-k_{1}^{2}} C_{b}(x) C_{2 a+b}(x)\right),
\end{gathered}
$$

where $k_{i}, \sqrt{w^{2}-k_{i}}, i=1,2,3$ are defined by (7).

\section{Types OF BOUNDARY CONDITIONS}

In this paper we consider three types of boundary conditions.

(1.) The first type, called a Dirichlet boundary condition, defines the value of the function itself:

$$
\Psi(\boldsymbol{x})=f(\boldsymbol{x}), \text { for } \boldsymbol{x} \in \partial F,
$$

where $f(\boldsymbol{x})$ is a given function defined on the boundary.

(2.) The second type, called a Neumann boundary condition, defines the value of the normal derivative of the function:

$$
\frac{\partial \Psi}{\partial \boldsymbol{n}}(\boldsymbol{x})=f(\boldsymbol{x}), \text { for } \boldsymbol{x} \in \partial F,
$$

where $\boldsymbol{n}$ denotes normal to the boundary $\partial F$. 


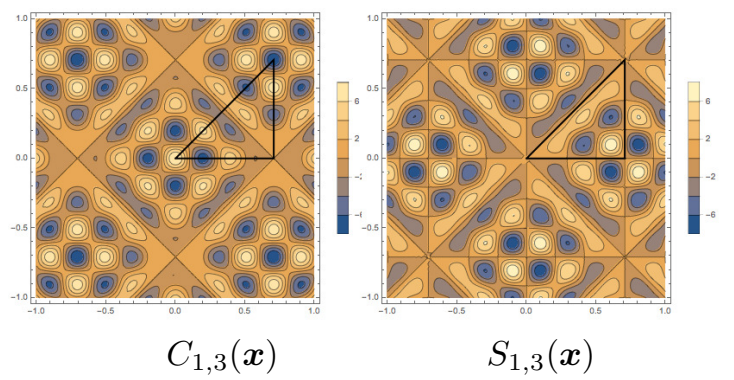

Figure 4. The contour plot of $C_{1,3}(\boldsymbol{x}), S_{1,3}(\boldsymbol{x})$. The triangle denotes the fundamental domain $F$ of the affine Weyl group $C_{2}$.

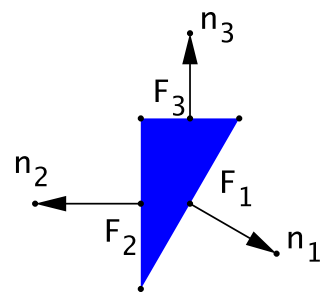

Figure 6. The normal vectors and boundaries are indicated for the Weyl group $G_{2}$

(3.) The third type, called a mixed boundary condition, defines the value of the function itself on one part of the boundary and the value of the normal derivative of the function on the other part of the boundary:

$$
\begin{cases}\Psi(\boldsymbol{x})=f_{0}(\boldsymbol{x}) & \text { for } \boldsymbol{x} \in \partial F_{0}, \\ \frac{\partial \Psi}{\partial \boldsymbol{n}}(\boldsymbol{x})=f_{1}(\boldsymbol{x}) & \text { for } \boldsymbol{x} \in \partial F_{1},\end{cases}
$$

where $\partial F=\partial F_{0} \cup \partial F_{1}$ and $f_{0}(\boldsymbol{x}), f_{1}(\boldsymbol{x})$ are given functions, defined on the appropriate boundary.

Remark 4 [12]. For the Dirichlet boundary conditions all eigenvalues are positive.

For the Neumann boundary condition all eigenvalues are non-negative.

In Table 1 we present how the four types of functions, defined in Section 3 behave on the boundary $\partial F$ of the fundamental region $F$. The fundamental region $F$ for $C_{2}$ and $G_{2}$ groups is presented in Figure 2. Symbol $s$ corresponds to the reflection orthogonal to the short root and $l$ corresponds to the reflection orthogonal to the long root.

\section{1. $C_{2}$ CASE}

The normal vectors to the fundamental region $F$ of the Weyl group $C_{2}$ are the following:

$$
\boldsymbol{n}_{1}=(0,-1)_{e}, \quad \boldsymbol{n}_{2}=\left(-\frac{1}{\sqrt{2}}, \frac{1}{\sqrt{2}}\right)_{e}, \quad \boldsymbol{n}_{3}=(1,0)_{e} .
$$

In Figure 3 we present the fundamental region $F$ with indicated boundaries and corresponding normal vectors.

The values of the four families of special functions $C, S, S^{s}$ and $S^{l}$ satisfying the Dirichlet boundary condition $(\mathbf{D})$ on the boundary $\partial F$ of the fundamental

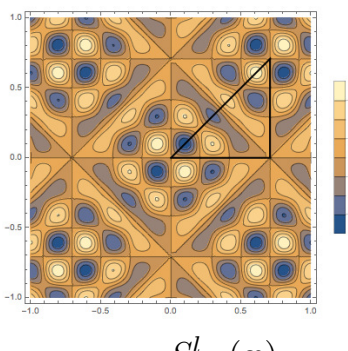

$$
S_{1,3}^{l}(\boldsymbol{x})
$$

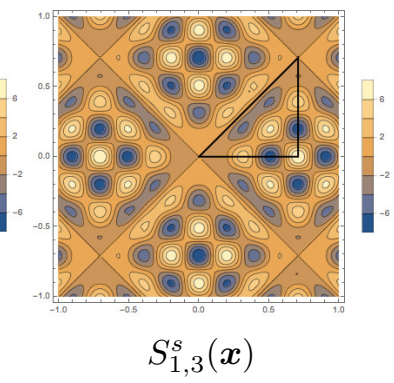

Figure 5. The contour plot of $S_{1,3}^{l}(\boldsymbol{x}), S_{1,3}^{s}(\boldsymbol{x})$. The triangle denotes the fundamental domain $F$ of the affine Weyl group $C_{2}$.

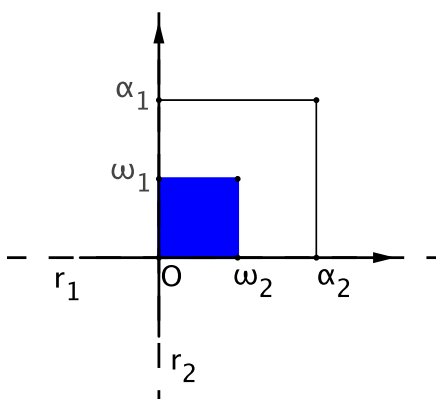

Figure 7. A shaded square represents the fundamental region $F$ of $A_{1} \times A_{1}$.

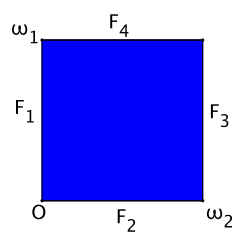

Figure 8 . The Boundaries of the fundamental region $F$ of $A_{1} \times A_{1}$ are indicated.

region $F$ are presented in Tables 2, 4, and 5. Tables 3 5 present the values of the functions satisfying the Neumann boundary condition $(\sqrt{\mathbf{N}})$. The examples of functions and their behaviours on the boundary $\partial F$ is presented in Figures 4 and 5 .

\section{2. $G_{2}$ CASE}

The normal vectors to the fundamental region $F$ of the Weyl group $G_{2}$ are the following:

$$
\boldsymbol{n}_{1}=(-1,0)_{e}, \quad \boldsymbol{n}_{2}=\left(\frac{\sqrt{3}}{2},-\frac{1}{2}\right)_{e}, \quad \boldsymbol{n}_{3}=\left(\frac{1}{2}, \frac{\sqrt{3}}{2}\right)_{e}
$$

In Figure 6 we present the fundamental region $F$ with indicated boundaries and corresponding normal vectors.

The values of the functions for group $G_{2}$ fulfilling the Dirichlet boundary condition $(\mathbf{D}$ ) on the boundary of the fundamental region $F$ are presented in Tables 6, 8 and 9. The values of the functions satisfying the Neumann boundary condition $(\bar{N})$ are given in Tables 7,9. The examples of functions and their behaviours on the boundary $\partial F$ is presented in Figures 9 and 10 . 


\begin{tabular}{cc}
\hline$C_{a, b}(\boldsymbol{x})$ & Dirichlet condition \\
\hline$F_{1}$ & $2 C_{a+b}(x)+2 C_{b}(x)$ \\
\hline$F_{2}$ & $2 C_{a+b}(x) C_{b}(x)$ \\
\hline$F_{3}$ & $C_{a+b}(1) C_{b}(y)+C_{b}(1) C_{a+b}(y)$ \\
\hline
\end{tabular}

TABLE 2. Values of $C_{a, b}(\boldsymbol{x})$ function at the boundary of the fundamental region $F$ in $C_{2}$ case.

\begin{tabular}{cc}
\hline$S_{a, b}(\boldsymbol{x})$ & Neumann condition \\
\hline$F_{1}$ & $2 i\left(k S_{b}(x)-\sqrt{w^{2}-k^{2}} S_{a+b}(x)\right)$ \\
\hline$F_{2}$ & $2 i\left(\sqrt{w^{2}-k^{2}} S_{a+b}(x) C_{b}(x)-k S_{b}(x) C_{a+b}(x)\right)$ \\
\hline$F_{3}$ & $i\left(\sqrt{w^{2}-k^{2}} C_{a+b}(1) S_{b}(y)-k C_{b}(1) S_{a+b}(y)\right)$ \\
\hline
\end{tabular}

TABLE 3 . Values of $S_{a, b}(\boldsymbol{x})$ function at the boundary of the fundamental region $F$ in $C_{2}$ case.

\begin{tabular}{ccc}
\hline & \multicolumn{2}{c}{ Mixed condition } \\
\cline { 2 - 3 }$S_{a, b}^{s}(\boldsymbol{x})$ & Dirichlet condition & Neumann condition \\
\hline$F_{1}$ & $2\left(C_{a+b}(x)-C_{b}(x)\right)$ & 0 \\
\hline$F_{2}$ & 0 & $2 i\left(-\sqrt{w^{2}-k^{2}} S_{a+b}(x) C_{b}(x)+k S_{b}(x) C_{a+b}(x)\right)$ \\
\hline$F_{3}$ & $C_{(a+b)}(1) C_{b}(y)-C_{b}(1) C_{a+b}(y)$ & 0 \\
\hline
\end{tabular}

TABLE 4. Values of $S_{a, b}^{s}(\boldsymbol{x})$ function at the boundary of the fundamental region $F$ in $C_{2}$ case.

\begin{tabular}{ccc}
\hline & \multicolumn{2}{c}{ Mixed condition } \\
\cline { 2 - 3 }$S_{a, b}^{l}(\boldsymbol{x})$ & Dirichlet condition & Neumann condition \\
\hline$F_{1}$ & 0 & $2 i\left(-\sqrt{w^{2}-k^{2}} S_{b}(x)-k S_{a+b}(x)\right)$ \\
\hline$F_{2}$ & $2 S_{a+b}(x) S_{b}(x)$ & 0 \\
\hline$F_{3}$ & 0 & $i\left(\sqrt{w^{2}-k^{2}} C_{a+b}(1) S_{b}(y)+k C_{b}(1) S_{a+b}(y)\right)$ \\
\hline
\end{tabular}

TABLE 5. Values of $S_{a, b}^{l}(\boldsymbol{x})$ function at the boundary of the fundamental region $F$ in $C_{2}$ case.

\begin{tabular}{cc}
\hline$C_{a, b}(\boldsymbol{x})$ & Dirichlet condition \\
\hline$F_{1}$ & $2\left(C_{b}(y)+C_{3 a+b}(y)+C_{3 a+2 b}(y)\right)$ \\
\hline$F_{2}$ & $C_{2 a+b}(x) C_{b}(x)+C_{a+b}(x) C_{3 a+b}(x)+C_{a}(x) C_{3 a+2 b}(x)$ \\
\hline$F_{3}$ & $C_{2 a+b}(x) C_{b}(x-1)+C_{a+b}(x) C_{3 a+b}(x-1)+C_{a}(x) C_{3 a+2 b}(x-1)$ \\
\hline
\end{tabular}

TABLE 6 . Values of $C_{a, b}(\boldsymbol{x})$ function at the boundary of the fundamental region $F$ in $G_{2}$ case.

\begin{tabular}{cc}
\hline$S_{a, b}(\boldsymbol{x})$ & Neumann condition \\
\hline$F_{1}$ & $2 i\left(-k_{3} S_{3 a+2 b}(y)+k_{2} S_{3 a+b}(y)-k_{1} S_{b}(y)\right)$ \\
\hline$F_{2}$ & $-2 i\left(\sqrt{w^{2}-k_{3}^{2}} C_{3 a+2 b}(x) S_{a}(x)-\sqrt{w^{2}-k_{2}^{2}} C_{3 a+b}(x) S_{a+b}(x)+\sqrt{w^{2}-k_{1}^{2}} C_{b}(x) S_{2 a+b}(x)\right)$ \\
\hline$F_{3}$ & $2 i\left(k_{3} S_{3 a+2 b}(x-1) C_{a}(x)-k_{2} S_{3 a+b}(x-1) C_{a+b}(x)+k_{1} S_{b}(x-1) C_{2 a+b}(x)\right)$ \\
\hline
\end{tabular}

TABLE 7 . Values of $S_{a, b}(\boldsymbol{x})$ function at the boundary of the fundamental region $F$ in $G_{2}$ case. 


\begin{tabular}{ccc}
\hline & \multicolumn{2}{c}{ Mixed condition } \\
\cline { 2 - 3 }$S_{a, b}^{s}(\boldsymbol{x})$ & Dirichlet condition & Neumann condition \\
\hline$F_{1}$ & $2\left(-S_{b}(x)-S_{3 a+b}(x)+S_{3 a+2 b}(x)\right)$ & 0 \\
\hline & 0 & $2 i\left(\sqrt{w^{2}-k_{3}^{2}} C_{3 a+2 b}(x) C_{a}(x)\right.$ \\
$F_{2}$ & & $+\sqrt{w^{2}-k_{2}^{2}} C_{3 a+b}(x) C_{a+b}(x)$ \\
& & $\left.+\sqrt{w^{2}-k_{1}^{2}} C_{b}(x) C_{2 a+b}(x)\right)$ \\
\hline \multirow{2}{*}{$F_{3}$} & $-C_{2 a+b}(x) S_{b}(x-1)-C_{a+b}(x) S_{3 a+b}(x-1)$ & \\
& $+C_{a}(x) S_{3 a+2 b}(x-1)$ & \\
\hline
\end{tabular}

TABLE 8. Values of $S_{a, b}^{s}(\boldsymbol{x})$ function at the boundary of the fundamental region $F$ in $G_{2}$ case.

\begin{tabular}{ccc}
\hline & \multicolumn{2}{c}{ Mixed condition } \\
\cline { 2 - 3 }$S_{a, b}^{l}(\boldsymbol{x})$ & Dirichlet condition & Neumann condition \\
\hline$F_{1}$ & 0 & $2 i\left(-k_{3} C_{3 a+2 b}(y)-k_{2} C_{3 a+b}(y)+k_{1} C_{b}(y)\right)$ \\
\hline \multirow{2}{*}{$F_{2}$} & $-S_{2 a+b}(x) C_{b}(x)+S_{a+b}(x) C_{3 a+b}(x)$ & 0 \\
\hline & $+S_{a}(x) C_{3 a+2 b}(x)$ & $2 i\left(k_{3} C_{3 a+2 b}(x-1) C_{a}(x)+k_{2} C_{3 a+b}(x-1) C_{a+b}(x)\right.$ \\
$F_{3}$ & 0 & $\left.-k_{1} C_{b}(x-1) C_{2 a+b}(x)\right)$ \\
\hline
\end{tabular}

TABLE 9 . Values of $S_{a, b}^{l}(\boldsymbol{x})$ function at the boundary of the fundamental region $F$ in $G_{2}$ case.
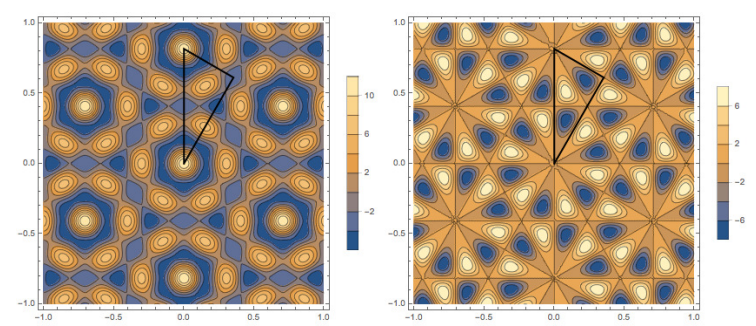

$\Re C_{1,3}(\boldsymbol{x})$

FIgURE 9. The contour plot of real part of $C_{1,3}(\boldsymbol{x})$, $S_{1,3}(\boldsymbol{x})$ functions. The triangle denotes the fundamental domain $F$ of the affine Weyl group $G_{2}$.

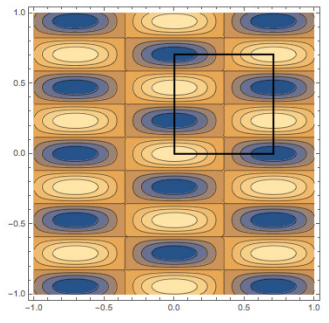

$\Re C_{1,3}(\boldsymbol{x})$

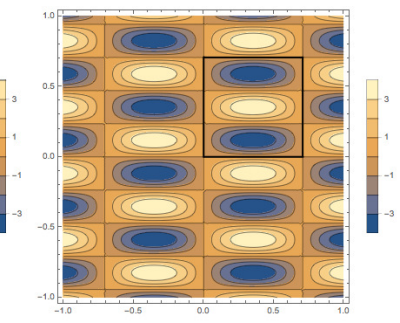

$\Re S_{1,3}(\boldsymbol{x})$

Figure 11. The contour plot of real part of $C_{1,3}(\boldsymbol{x})$, and $S_{1,3}(\boldsymbol{x})$ functions. The square denotes the fundamental domain $F$ of $A_{1} \times A_{1}$.

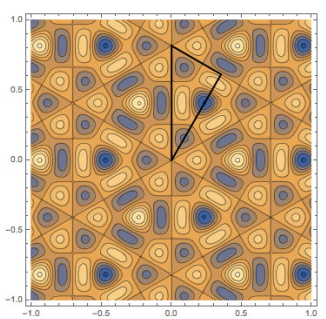

$$
\Im S_{1,3}^{l}(\boldsymbol{x})
$$

Figure 10. The contour plot of imaginary part of $S_{1,3}^{l}(\boldsymbol{x}), S_{1,3}^{s}(\boldsymbol{x})$ functions. The triangle denotes the fundamental domain $F$ of the affine Weyl group $G_{2}$.
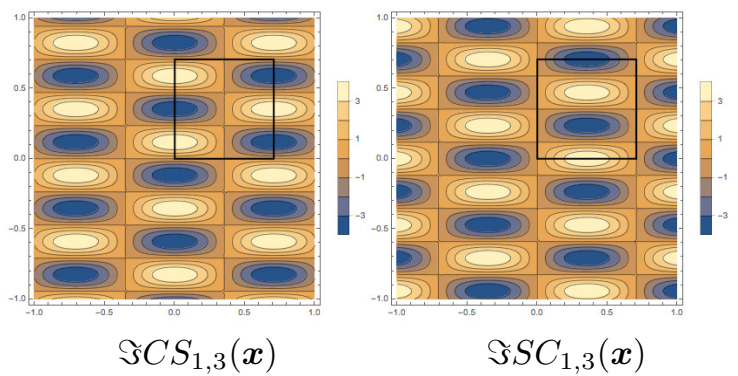

Figure 12. The contour plot of imaginary part of $C S_{1,3}(\boldsymbol{x})$, and $S C_{1,3}(\boldsymbol{x})$ functions. The square denotes the fundamental domain $F$ of $A_{1} \times A_{1}$. 


\begin{tabular}{cc}
\hline$C_{a, b}(\boldsymbol{x})$ & Dirichlet condition \\
\hline$F_{1}$ & $2 C_{b}(y)$ \\
\hline$F_{2}$ & $2 C_{a}(x)$ \\
\hline$F_{3}$ & $C_{a}(1) C_{b}(y)$ \\
\hline$F_{4}$ & $C_{a}(x) C_{b}(1)$ \\
\hline
\end{tabular}

TABLE 10 . Values of $C_{a, b}(\boldsymbol{x})$ function at the boundary $\partial F$ of the fundamental region $F$.

\begin{tabular}{ccc}
\hline & \multicolumn{2}{c}{ Mixed condition } \\
\cline { 2 - 3 }$C S_{a, b}(\boldsymbol{x})$ & Dirichlet & Neumann \\
\hline$F_{1}$ & $2 S_{b}(y)$ & 0 \\
\hline$F_{2}$ & 0 & $-2 i \sqrt{w^{2}-k^{2}} C_{a}(x)$ \\
\hline$F_{3}$ & $C_{a}(1) S_{b}(y)$ & 0 \\
\hline$F_{4}$ & 0 & $i \sqrt{w^{2}-k^{2}} C_{a}(x) C_{b}(1)$ \\
\hline
\end{tabular}

TABLE 12. Values of $C S_{a, b}(\boldsymbol{x})$ function at the boundary $\partial F$ of the fundamental region $F$.

\section{APPENDIX}

In this section we present the simplest case, namely $A_{1} \times A_{1}$. The fundamental region $F$ (shown in Figure 77 is a square with vertices $\left\{0, \omega_{1}, \omega_{2}, \omega_{1}+\omega_{2}\right\}$ in the $\omega$-basis.

The bases written in the orthonormal basis $\left\{e_{1}, e_{2}\right\}$ have the form

$$
\alpha_{i}=\sqrt{2} e_{i}, \quad \omega_{i}=\frac{1}{\sqrt{2}} e_{i} \quad i=1,2 .
$$

There are four families of special functions, namely $C$-, $S$-, $C S$ - and $S C$-functions. Their forms are the following:

$$
\begin{gathered}
C_{a, b}(\boldsymbol{x})=C_{a}(x) C_{b}(y)=4 \cos (2 \pi a x) \cos (2 \pi b y), \\
S_{a, b}(\boldsymbol{x})=S_{a}(x) S_{b}(y)=-4 \sin (2 \pi a x) \sin (2 \pi b y), \\
C S_{a, b}(\boldsymbol{x})=C_{a}(x) S_{b}(y)=4 i \cos (2 \pi a x) \sin (2 \pi b y), \\
S C_{a, b}(\boldsymbol{x})=S_{a}(x) C_{b}(y)=4 i \sin (2 \pi a x) \cos (2 \pi b y),
\end{gathered}
$$

where

$$
\begin{aligned}
& C_{\mu}(x)=e^{2 \pi i \mu x}+e^{-2 \pi i \mu x}, \\
& S_{\mu}(x)=e^{2 \pi i \mu x}-e^{-2 \pi i \mu x} .
\end{aligned}
$$

The behaviour of $C$-, $S$-, $C S$-, and $S C$-functions on the boundary $\partial F$ of the fundamental region $F$ described in Figure 8 is gathered in Tables 10,13 . The values if the functions we write using a separation constant $-k^{2}$ :

$$
-k^{2}=-2 a^{2} \pi^{2}, \quad w^{2}-k^{2}=2 b^{2} \pi^{2} .
$$

In Figures 11 and 12 plots of real and imaginary part of functions with weight $\lambda=(1,3)$ are shown.

\section{ACKNOWLEDGEMENTS}

The authors would like to thank Dr. J. Patera for stimulating discussions and comments.

\begin{tabular}{cc}
\hline$S_{a, b}(\boldsymbol{x})$ & Neumann condition \\
\hline$F_{1}$ & $-2 i k S_{b}(y)$ \\
\hline$F_{2}$ & $-2 i \sqrt{w^{2}-k^{2}} S_{a}(x)$ \\
\hline$F_{3}$ & $i k C_{a}(1) S_{b}(y)$ \\
\hline$F_{4}$ & $i \sqrt{w^{2}-k^{2}} S_{a}(x) C_{b}(1)$ \\
\hline
\end{tabular}

TABLE 11 . Values of $S_{a, b}(\boldsymbol{x})$ function at the boundary $\partial F$ of the fundamental region $F$.

\begin{tabular}{ccc}
\hline & \multicolumn{2}{c}{ Mixed condition } \\
\cline { 2 - 3 }$S C_{a, b}(\boldsymbol{x})$ & Dirichlet & Neumann \\
\hline$F_{1}$ & 0 & $-2 i k C_{b}(y)$ \\
\hline$F_{2}$ & $2 S_{a}(x)$ & 0 \\
\hline$F_{3}$ & 0 & $i k C_{a}(1) C_{b}(y)$ \\
\hline$F_{4}$ & $S_{a}(x) C_{b}(1)$ & 0 \\
\hline
\end{tabular}

TABLE 13. Values of $S C_{a, b}(\boldsymbol{x})$ function at the boundary $\partial F$ of the fundamental region $F$.

\section{REFERENCES}

[1] N. Bourbaki, Groupes et algèbres de Lie, Chapters IV, V, VI, Hermann, Paris 1968.

[2] D.J. Griffiths, R. College, Introduction to electrodynamics, Prentice Hall, New Jersey, 1999.

[3] J. E. Humphreys, Introduction to Lie Algebras and Representation Theory, New York, Springer, 1972.

[4] C. Jung, An exactly soluble three-body problem in onedimension, Vol. 58, Can. J. Phys., 58 (1980), 719-728.

[5] A. Klimyk, J. Patera, Orbit functions, SIGMA (Symmetry, Integrability and Geometry: Methods and Applications) 2 (2006), 006, 60 pages, math-ph/0601037.

[6] F.W. Lemire, J. Patera, M. Szajewska, Dominant weight multiplicities in hybrid characters of $B_{n}, C_{n}, F_{4}$, $G_{2}$, Internat. J. Theoret. Phys., vol. 54 (11) (2015), 4011-4026.

[7] W. Miller, Symmetry and separation of variables, with a foreword by Richard Askey, Encyclopedia of Mathematics and its Applications 4, Addison-Wesley Publishing Co., Reading, Mass.-London-Amsterdam, 1977.

[8] P. Moon, D.E. Spencer, Field Theory Handbook, Including Coordinate Systems, Differential Equations, and Their Solutions, 2nd ed. New York: Springer-Verlag, 1988.

[9] L. Motlochova, J. Patera, Four families of orthogonal polynomials of $C_{2}$ and symmetric and antisymmetric generalizations of sine and cosine functions, eprint arXiv:1101.3597.

[10] R.V. Moody, L. Motlochova, J. Patera, Gaussian cubature arising from hybrid characters of simple Lie groups, J. Fourier Analysis and its Applications, Online ISSN 1531-5851 (2014), DOI 10.1007/s00041-014-9355-0, 23 pp., arXiv:1202.4415. 
[11] M. Nesterenko, J. Patera, M. Szajewska, A. Tereszkiewicz, Orthogonal polynomials of compact simple Lie groups: Branching rules for polynomials, J. Phys. A Math. Theor. 43 (2010), no. 495207, 1-27.

[12] P. Olver, Introduction to Partial Differential Equations, Springer International Publishing, 2014.

[13] M. Szajewska, Four types of special functions of $G_{2}$ and their discretization, Integral Transform. Spec. Funct. vol. 23 (6) (2012), 455-472.
[14] M. Szajewska, Special functions and orthogonal polynomials of compact simple Lie groups and some of their applications, Warsaw University of Technology, Faculty of Mathematics and Information Science, 2011.

[15] A.N. Tikhonov, A.A. Samarskii, Equations of Mathematical Physics, Dover Publ., New York, 1990.

[16] S. Timoshenko, J.N. Goodier, Theory of Elasticity, McGraw-Hill Book Company, Inc., New York, 1961. 